\title{
Avons-nous percé le mystère de la globozoospermie ?
}

\section{Have we unravelled the mystery of globozoospermia?}

\author{
F. Ferfouri - G. Merdassi · F. Vialard \\ Reçu le 2 août 2011 ; accepté le 26 septembre 2011 \\ (C) SALF et Springer-Verlag France 2011
}

Résumé Si la première description de la globozoocéphalie date de 1971, il aura fallu plus de 35 ans pour identifier la première cause génétique (une altération du gène SPATA16), et cela malgré la création de nombreux modèles murins et la certitude d'une cause purement génétique à ce syndrome par l'observation de fratrie de patients atteints. Malgré l'identification récente de nouveaux gènes comme PICK1 et DPY19L2, la globozoocéphalie reste toujours un mystère. En effet, ce syndrome est probablement très polymorphe, comme le suggèrent les observations en microscopie électronique, et il est associé à un très mauvais taux de succès en assistance médicale à la procréation (AMP). Les prochaines études devront donc s'attacher à comprendre les mécanismes à l'origine de cette globozoocéphalie et à identifier des traitements pouvant contourner les déficits spermatiques majeurs associés à ce syndrome. Pour citer cette revue : Andrologie 21 (2011).

\section{Mots clés Globozoocéphalie · Génétique · Globozoospermie}

\begin{abstract}
Globozoocephaly was first described in 1971, and 35 years were necessary to identify the first genetic origin (SPATA16 mutation), despite the development of many mice models and the certainty of a genetic origin for this syndrome taking into account observations in many siblings. Despite the recent identification of the new genes PICK1 and DPY19L2, globozoocephaly is still a mystery. This syn-
\end{abstract}

F. Ferfouri $\cdot$ F. Vialard $(\bowtie)$

Laboratoire d'histologie, embryologie,

biologie de la reproduction, cytogénétique et génétique médicale,

CHI Poissy-Saint-Germain, France

e-mail : fvialard@hotmail.com

EA 2493, université Versailles-Saint-Quentin-en-Yvelines,

France

\section{G. Merdassi}

Unité de procréation médicalement assistée,

hôpital Aziza-Othmana, place de La Kasbah,

1008 Bab Menara Tunis, Tunisie drome is probably polymorphic, as suggested by electronic microscopic details, and it is associated with a poor success rate in assisted reproductive tehnology (ART). Further studies are needed to understand the mechanism leading to the globozoocephalia and to define new therapeutic approaches to turn around major spermatic defects associated with this syndrome. To cite this journal: Andrologie 21 (2011).

Keywords Globozoocephalia · Genetics · Globozoospermia

La globozoocéphalie spermatique a été décrite pour la première fois en 1971, et sa fréquence a été initialement estimée inférieure à $0,05 \%$ [1], puis ultérieurement réajustée à $0,1 \%$ chez les patients infertiles [2]. Le terme « globozoospermie » a été pour la première fois suggéré par Wolf et al. en 1976 [3]. Dans un sperme de patients fertiles, la fréquence des spermatozoïdes présentant une globozoocéphalie est évaluée inférieure à $0,5 \%$, et estimée à $2,3 \%$ dans le sperme de patients infertiles [4]. La globozoocéphalie est caractérisée par des spermatozoïdes présentant une tête ronde avec un acrosome absent ou anormal $[3,5,6]$. Cette anomalie s'associe fréquemment à une désorganisation de la pièce intermédiaire et du flagelle. Le spermogramme de ces patients montre donc une numération et une mobilité spermatique en général normale, associée à une altération majeure de la morphologie. Cette globozoocéphalie peut toucher l'ensemble des spermatozoïdes de l'éjaculat quand elle est totale, ou une fraction des spermatozoïdes de l'éjaculat lorsqu'elle est partielle.

Au total, l'absence d'acrosome est à l'origine d'une incapacité de ces spermatozoïdes à traverser la zone pellucide. Seul le recours à la fécondation in vitro avec injection intracytoplasmique du spermatozoïde (FIV-ICSI) permet à ces couples d'espérer avoir une descendance.

Dès les années 1970, l'étude de fratries de patients présentant une globozoocéphalie a fait émettre l'hypothèse d'une cause génétique pour expliquer la survenue de cette anomalie. Le développement de nombreux modèles murins a permis l'identification de mâles infertiles présentant des anomalies spermatiques dont la glogozoocéphalie. Le 
premier modèle murin de cette anomalie a été décrit en 1986 [7] chez les souris mâles blind-sterile qui présentaient une anomalie majeure de la spermatogenèse avec une altération de la formation de l'acrosome. Ce syndrome murin est dû à l'inactivation du gène Csnk2a2 (casein kinase II alpha catalytic subunit 2) [8]. Actuellement, aucune mutation de ce gène n'a été observée dans l'espèce humaine. Dès lors, de façon épisodique, différents modèles murins ont été décrits, avec à chaque fois des altérations de la formation de l'acrosome. Ainsi, il a été identifié les gènes $\mathrm{Hrb}(\mathrm{HIV}-1 \mathrm{Rev}$ binding protein) [9], GOPC (Golgi-associated PDZ and coiled-coil motif containing protein) [10], $\beta$-glucosidase 2 [11], PICK1 (protein interacting with c kinase 1) [12] et récemment Hsp90b1 (heat-shock protein, 90-kd, $\beta$-member 1) [13]. Ces protéines ont, dans leur majorité, un rôle au niveau des granules proacrosomiques de l'appareil de Golgi durant la spermiogenèse.

Avec l'identification de mutations dans les modèles murins, il a été envisagé de rechercher des mutations chez l'homme [14]. Plusieurs équipes ont séquencé, chez quelques patients atteints, différents gènes comme Csnk2a2 et Csnk2b [15], Hrb, GOPC et Csnk2a2 [16]. Si initialement aucune mutation n'a été retrouvée, mis à part quelques polymorphismes génétiques, une étude récente, analysant les gènes GOPC, HRB, CSNK2a2 et PICK1, a caractérisé une mutation pour ce dernier gène [17]. À ce jour, aucune autre mutation sur ce gène n'a été rapportée.

Puis, avec le développement des techniques de génétique moléculaire, il a été possible de réaliser ce que l'on appelle vulgairement « un tour de génome », c'est-à-dire étudier à l'aide des puces génomiques plusieurs milliers, voire million, de locus et identifier chez un individu les pertes d'hétérozygotie. Ces pertes correspondent en général à des zones dites de « consanguinité » et sont très importantes pour rechercher des altérations géniques dans les familles de patients atteints d'une même pathologie. C'est ce qui a été réalisé chez une famille syrienne où, sur une fratrie de six frères, trois ont présenté une globozoocéphalie [18]. Il a été identifié ainsi le gène SPATA16, muté chez les individus atteints. Ce gène code pour une protéine de l'appareil de Golgi, comme les gènes précédemment cités, et sa localisation est importante au niveau des vésicules proacrosomiques. Néanmoins, depuis 2007, aucune autre famille de patients présentant une globozoocéphalie n'a été rapportée, et il est possible que les altérations du gène SPATA16 ne soient impliquées que dans un nombre restreint de familles.

Parallèlement, il a été réalisé une étude protéomique en comparant les protéines spermatiques issues de patients fertiles à celles des spermatozoïdes globozoocéphales d'un patient infertile [19]. Cette étude a permis de montrer une différence d'expression pour 35 protéines $(9$ surexprimées et 26 sous-exprimées) impliquées dans différents mécanismes comme le métabolisme, la mobilité spermatique, le cytosque- lette, la méiose masculine et la fusion gamétique. Cette étude présente néanmoins deux écueils. D'une part, elle ne s'appuie que sur un unique patient ayant une globozoocéphalie. D'autre part, elle n'étudie que le stade ultime de la maturation spermatique, le spermatozoïde, or les gènes précédemment décrits sont tous des gènes exprimés durant la spermiogenèse, et principalement dans l'appareil de Golgi qui est éliminé durant les derniers stades de la maturation.

Puis, en début d'année, il a été rapporté une délétion homozygote récurrente du gène DPY19L2 chez plusieurs patients présentant un globozoocéphalie homogène [20]. Sur les 19 patients étudiés, 15 présentaient une délétion homozygote de ce gène. L'identification de ce gène a été possible grâce à la réalisation d'un « tour de génome » qui a permis d'identifier des zones d'homozygotie. Ce gène a une action sur la polarité cellulaire avec une action probable sur la formation de l'acrosome et l'élongation de la tete spermatique. Cela en fait donc un excellent candidat pour expliquer la globozoocéphalie chez ces patients. Cette protéine n'est retrouvée que dans le testicule, aucune détection n'a pu être faite dans le sperme éjaculé.

L'analyse fine des séquences en amont et en aval de ce gène a permis de montrer que celui-ci était flanqué de part et d'autre de séquences répétées (LCR), à l'origine de recombinaison homologue déséquilibrée. Cela explique donc, chez des patients non apparentés, l'identification d'une délétion identique n'emportant que le gène DPY19L2. Cette délétion, à l'état hétérozygote, est d'ailleurs rapportée, dans les bases de données, comme un variant chromosomique sans impact sur les patients au niveau mental [21]. Sa fréquence a été estimée à $1 / 125$, ce qui fait une fréquence estimée de la délétion homozygote à $1 / 62500$. Cette délétion est très probablement la cause majeure de la globozoocéphalie dans l'espèce humaine, et explique également le peu de mutations rapportées jusqu'à présent. Néanmoins, comme la globozoocéphalie spermatique est d'une grande hétérogénéité phénotypique [22], ce dont suggèrent les observations en microscopie électronique, il existe probablement d'autres étiologies génétiques pour expliquer ce syndrome.

Afin de résoudre ce trouble spermatique, il a été proposé à ces patients le recours à la FIV-ICSI. Or, depuis 1994 et la naissance du premier enfant issu d'un parent ayant une globozoocéphalie [23], plusieurs publications ont été rapportées, mais les taux de fécondation et de grossesse restent faibles. Le taux de fécondation moyen est de 30,8\% (123/400) [22] avec pour ces couples un maximum à $65,0 \%$ (13/20) [24]. Le taux d'implantation embryonnaire moyen est de 20,0\% (19/95), et le taux de grossesse par tentative de 22,6\% (14/62), dont quatre fausses couches ou grossesses extra-utérines. Ainsi, si une cause majeure de la survenue de la globozoocéphalie a été identifiée, il reste à comprendre la raison pour laquelle le faible taux de succès en FIV-ICSI 
pour ces patients en comparaison aux taux observés en ICSI en général.

Afin de compenser ce déficit de fécondation, il a été proposé différentes méthodes d'activation ovocytaire avant de réaliser l'ICSI [22]. Les taux de fécondation moyens et de grossesse par tentative sont alors de 75,3\% (205/272) et $38,1 \%(8 / 21)$ respectivement, soit supérieurs à ceux obtenus en ICSI classique.

Puis, il a été montré que la globozoocéphalie pourrait être associée à un déficit en PLCzeta [25]. En se basant sur cette donnée et sur le fait que PLCzeta est à l'origine des oscillations calciques nécessaires à l'activation ovocytaire, il a été réalisé, chez un patient présentant une globozoocéphalie spermatique et un déficit en PLCzeta, une ICSI avec activation ovocytaire par du calcium ionophore, et une grossesse a été obtenue [26]. De la même façon, une ICSI a été réalisée avec le même succès en réalisant une activation électrique ovocytaire [27]. Si cette approche présente une avancée dans la prise en charge des patients ayant une globozoocéphalie, il reste néanmoins à garantir, pour ces couples, l'innocuité d'une telle approche.

Une autre cause des échecs de la FIV-ICSI pourrait être une altération quantitative ou qualitative de l'ADN spermatique. Différentes études ont été réalisées sur ce sujet, mais les résultats restent très contradictoires aussi bien dans l'augmentation du taux d'aneuploïdie spermatique que du taux de fragmentation de 1'ADN spermatique [28-30]. C'est la raison pour laquelle il a été très récemment proposé l'utilisation de l'IMSI, et une première grossesse vient d'être décrite [31]. D'autres cas devront être rapportés afin de savoir si cela pourra être appliqué à l'ensemble des patients globozoocéphales ou uniquement à un certain type.

D'autres études seront nécessaires pour élucider ce phénomène en se basant :

- sur une description fine de l'anomalie spermatique ;

- sur une analyse génétique avec, entre autres, la recherche de la délétion du gène DPY19L2.

En conclusion, si une grande avancée a été obtenue dans l'identification des déficits génétiques liés à la globozoocéphalie, il reste encore des travaux de recherche importants pour faire un lien entre ce phénotype spermatique, les déficits génétiques et les faibles taux de fécondation en FIV-ICSI. L'identification de ces mécanismes devrait nous permettre alors de proposer de nouvelles stratégies, peut-être moins invasives que celles proposées actuellement en utilisant du calcium ionophore ou une stimulation électrique. Au total, le mystère de la globozoocéphalie n'a pas encore été totalement percé, de nombreux travaux seront probablement encore nécessaires.

Conflit d'intérêt : les auteurs déclarent ne pas avoir de conflit d'intérêt.

\section{Références}

1. Schirren C, Holstein ASchirren C (1971) Uber die morphogenese rundkopfiger spermatozoen des menschen. Andrologia 3:117-25

2. Holstein AF, Schirren CG, Schirren C, Mauss J (1973) Round headed spermatozoa: a cause of male infertility. Dtsch Med Wochenschr 98:61-2

3. Wolff HH, Schill WB, Moritz P (1976) Round-headed spermatozoa: a rare andrologic finding ("globe-headed spermatozoa", "globozoospermia"). Hautarzt 27:111-6

4. Kalahanis J, Rousso D, Kourtis A, et al (2002) Round-headed spermatozoa in semen specimens from fertile and subfertile men. J Reprod Med 47:489-93

5. Pedersen H, Rebbe H (1974) Fine structure of round-headed human spermatozoa. J Reprod Fertil 37:51-4

6. Kullander S, Rausing A (1975) On round-headed human spermatozoa. Int J Fertil 20:33-40

7. Sotomayor RE, Handel MA (1986) Failure of acrosome assembly in a male sterile mouse mutant. Biol Reprod 34:171-82

8. Xu X, Toselli PA, Russell LD, Seldin DC (1999) Globozoospermia in mice lacking the casein kinase II alpha' catalytic subunit. Nat Genet 23:118-21

9. Kang-Decker N, Mantchev GT, Juneja SC, et al (2001) Lack of acrosome formation in Hrb-deficient mice. Science 294: 1531-3

10. Yao R, Ito C, Natsume Y, et al (2002) Lack of acrosome formation in mice lacking a Golgi protein, GOPC. Proc Natl Acad Sci USA 99:11211-6

11. Yildiz Y, Matern H, Thompson B, et al (2006) Mutation of betaglucosidase 2 causes glycolipid storage disease and impaired male fertility. J Clin Invest 116:2985-94

12. Xiao N, Kam C, Shen C, et al (2009) PICK1 deficiency causes male infertility in mice by disrupting acrosome formation. J Clin Invest 119:802-12

13. Audouard C, Christians E (2011) Hsp90beta1 knockout targeted to male germline: a mouse model for globozoospermia. Fertil Steril 95:1475-7.e1-e4

14. Truong BN, Moses EK, Armes JE, et al (2003) Searching for candidate genes for male infertility. Asian J Androl 5:137-47

15. Pirrello O, Machev N, Schimdt F, et al (2005) Search for mutations involved in human globozoospermia. Hum Reprod 20:1314-8

16. Christensen GL, Ivanov IP, Atkins JF, et al (2006) Identification of polymorphisms in the Hrb, GOPC, and Csnk2a2 genes in two men with globozoospermia. J Androl 27:11-5

17. Liu G, Shi QW, Lu GX (2010) A newly discovered mutation in PICK1 in a human with globozoospermia. Asian J Androl 12:556-60

18. Dam AH, Koscinski I, Kremer JA, et al (2007) Homozygous mutation in SPATA16 is associated with male infertility in human globozoospermia. Am J Hum Genet 81:813-20

19. Liao TT, Xiang Z, Zhu WB, Fan LQ (2009) Proteome analysis of round-headed and normal spermatozoa by 2 -D fluorescence difference gel electrophoresis and mass spectrometry. Asian J Androl 11:683-93

20. Harbuz R, Zouari R, Pierre V, et al (2011) A recurrent deletion of DPY19L2 causes infertility in man by blocking sperm head elongation and acrosome formation. Am J Hum Genet 88: 351-61

21. Shaikh TH, Gai X, Perin JC, et al (2009) High-resolution mapping and analysis of copy number variations in the human genome: a data resource for clinical and research applications. Genome Res 19:1682-90

22. Dam AH, Feenstra I, Westphal JR, et al (2007) Globozoospermia revisited. Hum Reprod Update 13:63-75 
23. Lundin K, Sjogren A, Nilsson L, Hamberger L (1994) Fertilization and pregnancy after intracytoplasmic microinjection of acrosomeless spermatozoa. Fertil Steril 62:1266-7

24. Kilani ZM, Shaban MA, Ghunaim SD, et al (1998) Triplet pregnancy and delivery after intracytoplasmic injection of roundheaded spermatozoa. Hum Reprod 13:2177-9

25. Yoon SY, Jellerette T, Salicioni AM, et al (2008) Human sperm devoid of PLC, zeta 1 fail to induce $\mathrm{Ca}(2+)$ release and are unable to initiate the first step of embryo development. J Clin Invest 118:3671-81

26. Taylor SL, Yoon SY, Morshedi MS, et al (2010) Complete globozoospermia associated with PLC zeta deficiency treated with calcium ionophore and ICSI results in pregnancy. Reprod Biomed Online 20:559-64

27. Egashira A, Murakami M, Haigo K, et al (2009) A successful pregnancy and live birth after intracytoplasmic sperm injection with globozoospermic sperm and electrical oocyte activation. Fertil Steril 92:2037.e5-e9

28. Vicari E, Perdichizzi A, De Palma A, et al (2002) Globozoospermia is associated with chromatin structure abnormalities: case report. Hum Reprod 17:2128-33

29. Brahem S, Mehdi M, Elghezal H, Saad A (2011) Analysis of sperm aneuploidies and DNA fragmentation in patients with globozoospermia or with abnormal acrosomes. Urology 77:1343-8

30. Perrin A, Louanjli N, Ziane Y, et al (2011) Study of aneuploidy and DNA fragmentation in gametes of patients with severe teratozoospermia. Reprod Biomed Online 22:148-54

31. Sermondade N, Hafhouf E, Dupont C, et al (2011) Successful childbirth after intracytoplasmic morphologically selected sperm injection without assisted oocyte activation in a patient with globozoospermia. Hum Reprod: epub Aug 19

\section{型 Springer \\ the language of science \\ springerlink.com}

\section{SpringerLink}

Access outstanding STM content with sophisticated search tools

Books, journals, reference works, protocols \& more

Over 4 million documents on one integrated platform More productive searches with Semantic Linking
VISIT TODAY! 